\section{The antimicrobial potential of Lactobacillus acidophilus on pathogenic bacteria causing diarrhea}

Rasha H. Bassyouni ${ }^{*}$, Mohammed Wasim Ahmed Nassar², Zeinab Abdelkhalek Ibrahim², Mohammed Saad Zaghloul Ahmed ${ }^{1}$

1 Medical Microbiology and Immunology Faculty of Medicine, Fayoum University, Egypt

2 Medical Microbiology and Immunology Faculty of Medicine, Cairo University, Egypt

*Corresponding author: rhb00@fayoum. edu.eg

Object: Lactobacillus acidophilus is a nonpathogenic member of gastrointestinal tract and it is widely used in fermented dairy products. This study aimed to assess the antimicrobial potential of two strains of $L$. acidophilus on some pathogenic bacteria frequently causing diarrhea or gastroenteritis.

Methods: The antibacterial activity cell free supernatant (CFS) of two control standard strains of $L$. acidophilus (L. acidophilus-la5 and L. acidophilus against five control standard strains of bacteria causing diarrhea; Enterotoxogenic Escherichia coli (ETEC), Enterohaemorrhagic E. coli $\mathrm{O}_{157}: \mathrm{H}_{7}(\mathrm{EHEC})$ Salmonella typhimurium Shigella flexeneri and Staphylococcus aureus were determined using agar well diffusion method. The sensitivity of the pathogenic bacteria to the CFS of each $L$. acidophilus in relation to time was determined by standard plate count .The antibiotic susceptibility tests of 20 antibiotics against tested organisms with and without CFS were assessed by disc diffusion method. The minimum inhibitory concentration (MIC) of ciprofloxacin with and without CFS was determined by tube dilution method.

Results: Both Lactobacilli strains decrease the colony count of tested strains by more than $90 \%$ after 60 min contact time. Both Lactobacilli strains significantly improve the antibacterial effect of tested antibiotiCS against ETEC, S. typhimurium and $S$. aureus, and $S$. flexeneri $(P<$ 0.05). The MIC of ciprofloxacin alone against all tested strains was $15.625 \mu \mathrm{g} / \mathrm{ml}$, while when combined with both Lactobacilli CFSs, the MIC decreased significantly to $0.488 \mu \mathrm{g} / \mathrm{ml}$ for ETEC, S. typhimurium, and S. flexeneri and to $0.977 \mu \mathrm{g} / \mathrm{ml}$ for EHEC and $S$. aureus $(P=0.000)$.
Corresponding author:

Dr. Rasha H. Bassyouni

$\equiv$ rhb00@fayoum.edu.eg 
Conclusion: living $L$. acidophilus strains could be used in prevention and treatment of diarrhea caused by certain bacterial pathogens, either in fermented milk/ yoghurt or as mediations.

Key words: Probiotic, L. acidophilus, Bacterial Diarrhea

\section{Introduction}

Infective bacterial diarrhea is a global health problem especially in young children in developing countries with rotavirus is the most common identified pathogen [1]. Many bacterial species were implicated as a cause of infective diarrhea as Salmonella spp., Campylobacter spp., Shiga toxin-producing $E$. coli 0157:H7 strain, Shigella spp., Vibrio spp.; and Yersinia spp. Other diarrheagenic $E$. coli in particular enterotoxigenic $E$. coli and enteroaggregative $E$. coli are increasingly being reported as causes of acute diarrhea [2].

Oral rehydration solution and antimicrobials are the main treatments for acute diarrhea. However, when diarrheal patients were given probiotics prior to or during hospitalization, a reduction in the frequency of diarrheal symptoms has been reported in both adults and children [1, 3].

Probiotics are preparations of living bacteria and yeasts that possess a beneficial health effect when administrated in adequate amounts [1, 4].They have been extensively studied for their beneficial effects in preventing and treating many conditions, including the treatment of lactose intolerance, traveller's diarrhea and the prevention and treatment of hospital acquired diarrhea[5,6]. Previous studies have evaluated the effect of Lactobacillus rhamnosus on colonization of rotavirus and found a potential antimicrobial effect against it [7]. It has been reported that most probiotics are well tolerated with rare adverse effect and can be safely used in patients with underlying chronic diseases or in those on immunosuppressive therapy [1].

Lactic acid bacteria are a group of Gram-positive bacilli and cocci occurring naturally in gastrointestinal tract, plants and fermented foods, such as dairy products, meat and alcoholic beverages [4]. Most probiotics commercially available today belong to the genera Lactobacillus and Bifidobacterium. They are the most important group of microorganism used in food fermentations. They inhibit food spoilage and pathogenic bacteria by producing antimicrobial substances such as lactic acid, hydrogen peroxide and bacteriocins [8]. Several mechanisms by which probiotics in vivo mediate their health benefits in the host; first, certain probiotics have antimicrobial activity and can exclude or inhibit pathogens; second, they can enhance the intestinal epithelial barrier; third, probiotic bacteria are believed to modulate the host immune response [4].

The aim of this study was to investigate the antimicrobial potential of two $L$. acidophilus strain on pathogenic bacteria frequently causing diarrhea or gastroenteritis with and without antibiotics.

\section{Materials and Methods}

\section{The test organisms}

Five standard strains of diarrhea causing bacteria; Enterotoxogenic E. coli (ETEC) (ATCC 25922), Enterohaemorrhagic E. coli $\mathrm{O}_{157}: \mathrm{H}_{7}$ (EHEC) (ATCC 51659), S. typhimurium (ATCC 25566), S. flexeneri(ATCC 
29903, CCM 4422) and S. aureus (ATCC 13565) were used in this study. The test organisms were grown in Brain Heart Infusion broth (Oxoid Ltd., Basingstoke, UK) and incubated at $35^{\circ} \mathrm{C}$ for $18 \mathrm{hrs}$. The concentrations of the organisms were determined by spectrophotometric method and standardized for all organisms at $10^{3} \mathrm{cfu} / \mathrm{ml}$ [9].

\section{Preparation of cell-free L. acidophilus culture supernatants}

Two standard strains of $L$. acidophilus (L. acidophilus-la5 (L1) and L. acidophilus (ATCC 4356, DSM 20079) (L2) were grown in MRS broth (Oxoid Ltd., Basingstoke, UK) at $35^{\circ} \mathrm{C}$ for $18 \mathrm{hrs}$ and adjusted to $0.5 \mathrm{McFarland}$. The cultures were centrifuged at $10000 x \mathrm{f}$ for $15 \mathrm{~min}$ and the resulted supernatant was designated crude cell -culture free supernatant (CFS). These supernatants were used immediately or stored at $-20^{\circ} \mathrm{C}$ until needed for antibacterial activity [10].

\section{Determination of the antibacterial activity of L. acidophilus}

The antibacterial effect of L1 and L2 was investigated by the following methods:

1. Agar well diffusion method (Qualitative inhibitory effect of CFS of lactobacilli strains). Agar well diffusion method was used as described by Wolf and Gibbones [11]. Briefly the freshly prepared inoculum $\left(10^{8} \mathrm{CFU} / \mathrm{mL}\right)$ was swabbed all over the surface of the Muller Hinton plate (Oxoid Ltd., Basingstoke, UK) using sterile cotton swab. Five wells were bored in the medium with the help of sterile corkborer having 5-mm diameter and were labeled properly. Then $50 \mu \mathrm{L}$ of each CFS was added to each well, $50 \mu \mathrm{L}$ of sterile nutrient broth was added to a well as a control. The experiment was done in triplicate. All plates were incubated at $37^{\circ} \mathrm{C}$ for 24 hours. After incubation, zones of inhibition (IZ) were measured.
2. Standard plate count (Quantitative inhibitory effect of CFS of lactobacilli strains): Equal amounts of CFS of each L. acidophilus and each test organism at $10^{3} \mathrm{CFU} / \mathrm{ml}$ were mixed in a sterile flask. The mixture was stirred gently. $100 \mu$ l was immediately transferred to nutrient agar ( 0 contact time) and incubated at $35^{\circ} \mathrm{C}$ for $18-24$ hours. $100 \mu$ sterile MRS broth was used as a negative control. This procedure was repeated at intervals of 10 minutes up to 60 minutes $(0,10,20,30,40,50,60$ minutes). Standard plate count was evaluated after incubation [9].

3. Antibiotic susceptibility test with and without lactobacilli. This test was done by using KirbyBauer disc diffusion method. Muller-Hinton plates were inoculated by swabbing the tested organism after dilution of $10^{8} \mathrm{cfu} / \mathrm{ml}$ organisms with equal amount of nutrient broth (Oxoid Ltd., Hampshire, UK) onto the surface of agar plates. Antibiotic discs were applied and plates were incubated for 24 hours at $35^{\circ} \mathrm{C}$. The antibacterial activities of antibiotics were assessed by measuring the inhibition zone (IZ) in $\mathrm{mm}$. Equal amounts of CFS of each $L$. acidophilus and each test organism at $10^{8} \mathrm{cfu} / \mathrm{ml}$ was mixed in a sterile flask at 0 contact time and the previous steps were performed and the results of both of tests were compared [12]. The following antibiotics discs (Oxoid Ltd., Hampshire, U K) were used: Rifampicin (30 $\mu \mathrm{g})$, Norfloxacin $(10 \mu \mathrm{g})$, Cefepime $(30 \mu \mathrm{g})$, Cefoperazone $(30 \mu \mathrm{g})$, Chloramphenicol (30 $\mu \mathrm{g})$, Ampicillin/Sulbactam $(20 \mu \mathrm{g})$, Amoxicillin $(10 \mu \mathrm{g})$, Ciprofloxacin $(5 \mu \mathrm{g})$, Gentamycin $(\mu \mathrm{g})$, Cefotraxione (CRO) with $30 \mu \mathrm{g}$ disc content, Sulphamethoxazole/Trimethprim $(25 \mu \mathrm{g})$, Doxycyline $(30 \mu \mathrm{g})$, Erythromycin $(15 \mu \mathrm{g})$, Amikacin $(30 \mu \mathrm{g})$, Amoxicillin/Clavulanic acid (30 $\mu \mathrm{g})$, Vancomycin $(30 \mu \mathrm{g})$, Meropenem $(10 \mu \mathrm{g})$, Clindamycin $(10 \mu \mathrm{g})$, Tobromycin $(30 \mu \mathrm{g})$, Cefotaxime $(30 \mu \mathrm{g})$. 
4. Antibacterial effect of L. acidophilus on minimum inhibitory concentration (MIC) of ciprofloxacin against diarrhea causing bacteria. MIC of ciprofloxacin on tested organisms with and without lactobacilli supernatant were performed using macro-tube dilution method. MICs of ciprofloxacin alone and MICs of CFS alone: for each organism was determined as described by Clinical and laboratory standards institute [13]. For determination of MIC of ciprofloxacin with lactobacilli, serial two-old dilution of ciprofloxacin in supernatant of each Lactobacillus CFS was prepared and inoculated with $25 \mu$ l of $10^{8} \mathrm{cfu} / \mathrm{ml}$ of test organism to each tube, mixed well and incubated at $35^{\circ} \mathrm{C}$ for $18 \mathrm{hrs}$

\section{Statistical analysis}

All statistical analysis were done using computer programs SPSS (Statistical Package for the Social Science; SPSS Inc., Chicago, IL, USA) version 15 for Microsoft Windows. The independent samples $t$-test was used for comparison between the antimicrobial effect of antibiotic with and without L1 and L2. P value $\leq 0.05$ was considered the cut-off value for statistically significance and $P$ value $\leq 0.001$ was considered highly significant.

\section{Results}

Both Lactobacilli (L1 and L2) strains dramatically reduced the colony count of tested strains; the decrease is directly proportional to the time of contact between organism and lactobacilli supernatants (Figure 1). We found more than 50\% reduction in colony count of all tested organisms after ten minutes of contact with supernatants of both Lactobacilli, except for $S$. aureus it needs $30 \mathrm{~min}$. The maximal effect was on ETEC. L2 needs more contact time than L1except for S. aureus. No significant difference between L1 and L2 supernatants in their antibacterial effect against tested organisms $(P \geq$ 0.05).

The inhibitory diameter of L1 and L2 against tested organisms are shown in Table 1. S.typhimurium is apparently the most affected organism although the differences between the two diffusion diameters of $\mathrm{L} 1$ and $\mathrm{L} 2$ are non-significant $(P>0.05)$.

Table 1. Inhibition zone ( $\mathrm{mm} \pm \mathrm{SD}$ ) of $\mathrm{L}$. acidophilus (L1 and L2) on tested organisms

\begin{tabular}{|c|c|c|}
\hline $\begin{array}{c}\text { Tested } \\
\text { organisms }\end{array}$ & $\begin{array}{c}\text { L. acidophilus-la5 } \\
\text { (L1) }\end{array}$ & $\begin{array}{c}\text { L. acidophilus } \\
\text { ATCC 4356(L2) }\end{array}$ \\
\hline EHEC & $12 \pm 1$ & $12 \pm 2$ \\
\hline ETEC & $16 \pm 2$ & $14 \pm 1$ \\
\hline S. typhimurium & $16 \pm 1$ & $19 \pm 2$ \\
\hline S. flexeneri & $15 \pm 1$ & $12 \pm 1$ \\
\hline S. aureus & $12 \pm 2$ & $14 \pm 1$ \\
\hline
\end{tabular}

Table 2 shows both Lactobacilli strains significantly improve the antibacterial effect of tested antibiotics by disc diffusion method against ETEC, S. typhimurium and $S$. aureus, and $S$. flexeneri $(P$ $<0.05$ ). It seems that the best effect of $L 1$ and $L 2$ were on amoxicillin/clavulanic acid, Cefoperazone, clindamycin, erythromycin, rifampicin and vancomycin.

L1 and L2 potentiate the antibacterial effect of ciprofloxacin on Enterotoxogenic E. coli, Enterohaemorrhagic E. coli 0157:H7, S. typhimurium, S. flexeneri and $S$. aureus as the MIC of ciprofloxacin alone against all tested strains was $15.625 \mu \mathrm{g} / \mathrm{ml}$, while when combined with L1 or L2 supernatant, the MIC significantly decreased to $0.48 \mu \mathrm{g} / \mathrm{ml}$, for ETEC, S. typhimurium, and S. flexeneri and to 0.977 $\mu \mathrm{g} / \mathrm{ml}$, for EHEC and S. aureus $(P=0.000)$ (Figure2).

Ciprofloxacin is effective against all bacteria tested, and showed almost similar results (inhibi- 


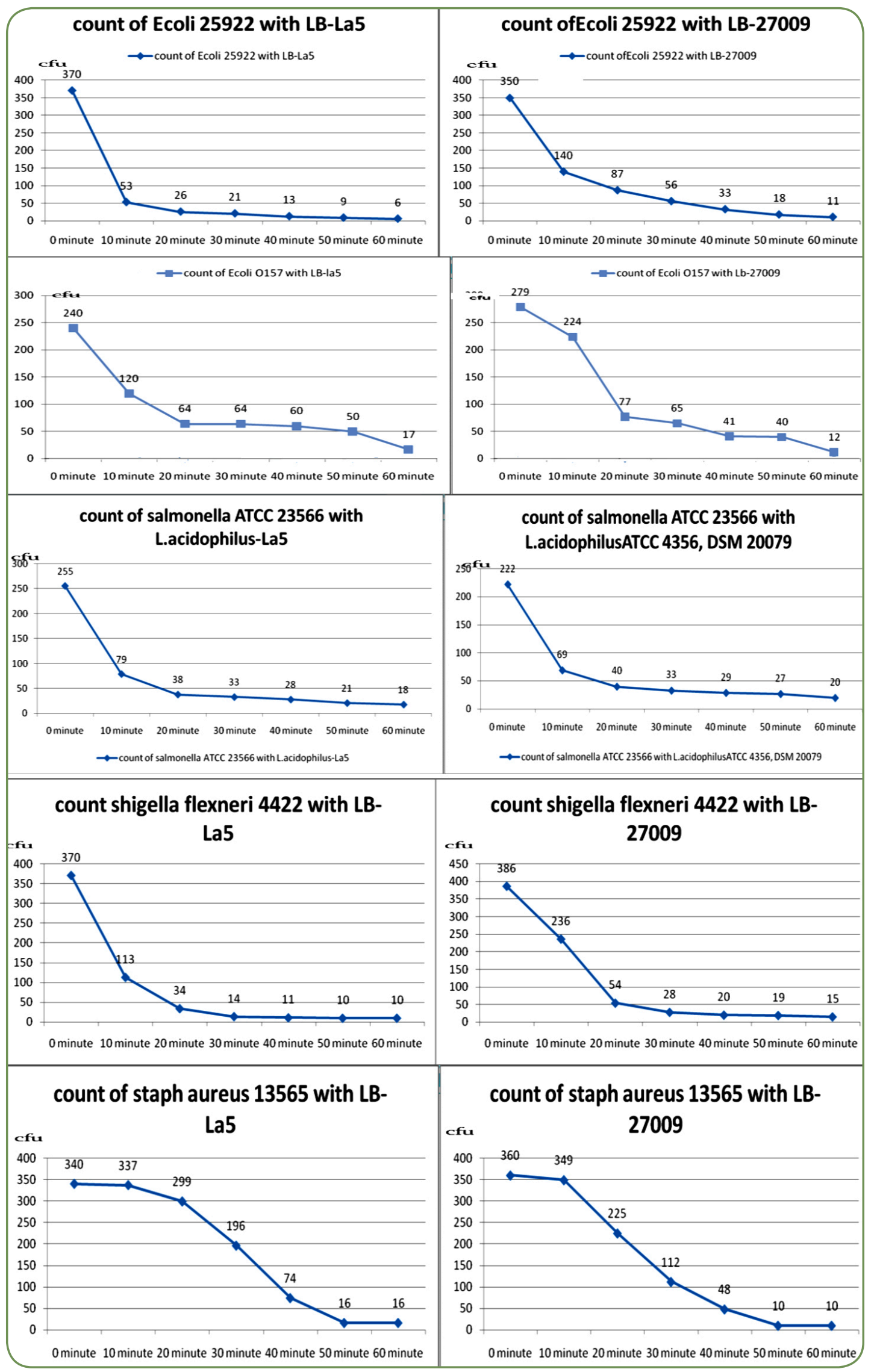

Figure 1. Time kill curve of L. acidophilus on tested organisms: 
Table 2. The antimicrobial effect of different antibiotics on tested organisms with and without L1 and L2 by disc diffusion methods ( $\mathrm{mm}$ )

\begin{tabular}{|c|c|c|c|c|c|c|c|c|c|c|c|c|c|c|c|}
\hline Antibiotic & EHEC & EHEC+L1 & EHEC+L2 & ETEC & ETEC+L1 & ETEC+L2 & S. typhimurium & S.typhimurium+L1 & typhimurium $+L 2$ & flexeneri & S. flexeneritL1 & S. flexeneritL2 & S. aureus & aureus $+L 1$ & $\overline{\text { S. aureus }+L 2}$ \\
\hline Amoxicillin & $0=R$ & $0=R$ & $0=R$ & $0=R$ & $0=R$ & $0=R$ & $12=R$ & $13=R$ & $12=R$ & $18=1$ & $19=1$ & $18=1$ & $9=R$ & $12=R$ & $13=R$ \\
\hline Ampicillin/Sulbactam & $0=R$ & $0=R$ & $0=R$ & $0=R$ & $0=R$ & $0=R$ & $0=R$ & $10=R$ & $0=R$ & $15=S$ & $18=S$ & $24=5$ & $17=S$ & $23=5$ & $22=5$ \\
\hline Amoxicillin/Clavulani & $12=R$ & $14=1$ & $15=1$ & $18=R$ & $21=R$ & $19=R$ & $17=1$ & $21=5$ & $21=S$ & $15=1$ & $16=1$ & $15=1$ & $10=R$ & $13=R$ & $16=R$ \\
\hline Cefepime & $15=1$ & $S=23$ & $25=S$ & $20=5$ & $26=5$ & $27=5$ & $0=R$ & $10=R$ & $11=R$ & $0=R$ & $0=R$ & $0=R$ & $16=1$ & $16=1$ & $17=1$ \\
\hline Cefoperazone & $20=1$ & $21=S$ & $21=5$ & $23=5$ & $28=5$ & $28=5$ & $25=5$ & $28=5$ & $25=S$ & $20=1$ & $25=S$ & $35=5$ & $15=R$ & $16=1$ & $15=R$ \\
\hline Cefotaxime & $24=5$ & $40=S$ & $38=5$ & $17=1$ & $19=1$ & $19=1$ & $5=R$ & $12=R$ & $12=R$ & $24=S$ & $40=S$ & $40=S$ & $23=5$ & $23=5$ & $23=5$ \\
\hline Cefotraxione & $20=1$ & $25=S$ & $25=S$ & $23=5$ & $31=5$ & $31=5$ & $12=R$ & $20=1$ & $12=R$ & $23=5$ & $37=5$ & $40=5$ & $23=5$ & $26=5$ & $27=S$ \\
\hline Vancomycin & $0=R$ & $19=S$ & $0=R$ & $0=R$ & $0=R$ & $12=R$ & $0=R$ & $22=5$ & $25=S$ & $17=5$ & $20=S$ & $20=5$ & $16=5$ & $17=5$ & $18=5$ \\
\hline Meropenem & $23=5$ & $25=5$ & $26=5$ & $35=5$ & $43=5$ & $44=5$ & $36=5$ & $48=S$ & $44=5$ & $21=5$ & $23=5$ & $32=5$ & $23=5$ & $23=5$ & $24=5$ \\
\hline Clindamycin & $0=R$ & $20=1$ & $25=S$ & $0=R$ & $9=R$ & $0=R$ & $0=R$ & $46=S$ & $23=5$ & $35=5$ & $44=5$ & $40=5$ & $21=S$ & $28=5$ & $26=5$ \\
\hline Chloramphenicol & $28=5$ & $28=5$ & $31=5$ & $26=5$ & $34=5$ & $44=5$ & $28=5$ & $32=5$ & $32=5$ & $25=5$ & $35=5$ & $25=5$ & $29=5$ & $30=5$ & $30=5$ \\
\hline Rifampicin & $13=R$ & $21=5$ & $16=R$ & $17=1$ & $48=5$ & $43=5$ & $16=R$ & $38=5$ & $43=5$ & $10=R$ & $20=5$ & $32=5$ & $R=15$ & $40=5$ & $38=5$ \\
\hline \begin{tabular}{|l|} 
Norfloxacin \\
\end{tabular} & $30=5$ & $30=5$ & $32=5$ & $35=5$ & $40=5$ & $40=5$ & $32=5$ & $40=S$ & $39=5$ & $30=5$ & $33=5$ & $40=5$ & $30=5$ & $33=5$ & $32=5$ \\
\hline Ciprofloxacin & $31=5$ & $31=5$ & $32=5$ & $35=5$ & $43=5$ & $41=5$ & $35=5$ & $38=5$ & $42=5$ & $30=5$ & $46=S$ & $46=5$ & $32=5$ & $34=5$ & $33=5$ \\
\hline Gentamycin & $20=5$ & $20=5$ & $21=5$ & $25=5$ & $28=5$ & $37=5$ & $21=5$ & $35=5$ & $28=5$ & $17=S$ & $25=S$ & $30=5$ & $21=S$ & $22=5$ & $22=5$ \\
\hline Tobromycin & $16=5$ & $22=5$ & $25=5$ & $15=5$ & $20=5$ & $21=5$ & $19=5$ & $28=5$ & $30=5$ & $21=5$ & $24=S$ & $21=5$ & $18=S$ & $20=5$ & $20=5$ \\
\hline Doxycyline & $18=1$ & $31=5$ & $23=5$ & $16=5$ & $23=5$ & $25=5$ & $23=5$ & $43=5$ & $38=5$ & $16=5$ & $24=S$ & $28=5$ & $20=5$ & $32=5$ & $30=5$ \\
\hline Erythromycin & $0=R$ & $18=1$ & $0=R$ & $10=R$ & $38=5$ & $38=5$ & $0=R$ & $25=5$ & $35=5$ & $11=R$ & $11=R$ & $25=5$ & $13=R$ & $32=5$ & $30=5$ \\
\hline Amikacin & $21=5$ & $26=5$ & $26=5$ & $21=5$ & $27=5$ & $33=5$ & $21=5$ & $24=5$ & $23=5$ & $20=5$ & $31=5$ & $30=5$ & $20=5$ & $24=5$ & $22=5$ \\
\hline Sulphamethoxazoler & $27=5$ & $32=5$ & $32=5$ & $25=5$ & $31=5$ & $34=5$ & $27=5$ & $35=5$ & $35=5$ & $24=S$ & $36=5$ & $46=5$ & $30=5$ & $32=5$ & $30=5$ \\
\hline \begin{tabular}{|l|} 
Pvalue \\
\end{tabular} & 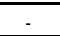 & 0.054 & 0.19 & . & 0.08 & $0.04^{*}$ & - & $0.003^{*}$ & $0.014^{*}$ & - & $0.036^{*}$ & $0.033^{*}$ & & $0.043^{*}$ & $0.046^{*}$ \\
\hline
\end{tabular}

R: Resistant, S: Susceptible, I: Intermediate resistant, *significant

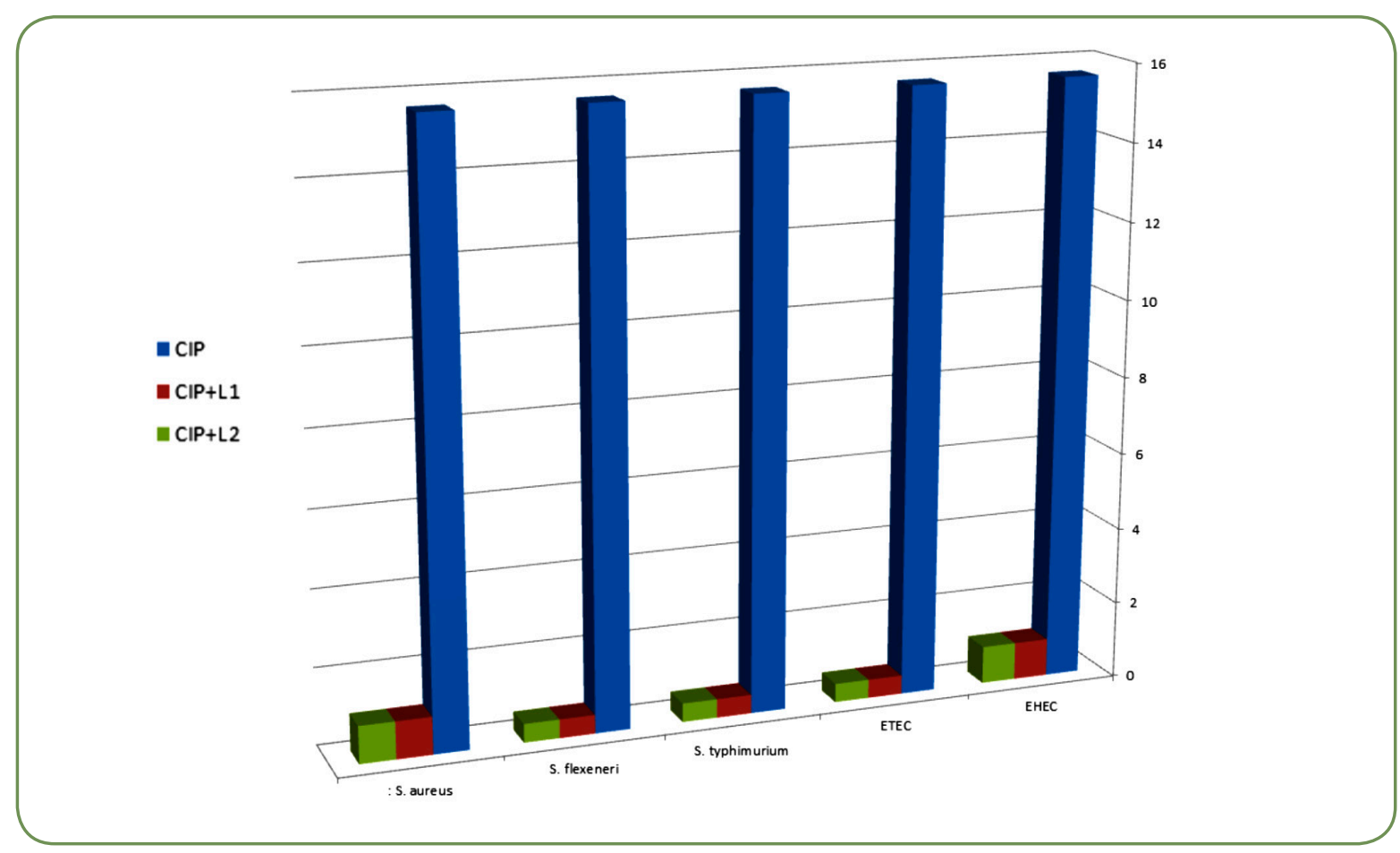

Figure 2. MICs of Ciprofloxacin ( $\mu \mathrm{g} / \mathrm{ml}$ ( with and without L. acidophilus ( $\mathrm{L} 1$ and L2) against tested organisms 
tion zone) with or without lactobacilli strains (L1 and L2) as shown in Table $\mathbf{2}$ and Figure 2. However, their MICs are highly different according to the method used and the difference in dilution factors. The inhibition zones of ETEC, S. typhimurium and S. flexeneri are considerably increased in presence of L1 and L2 with ciprofloxacin.

\section{Discussion}

We have studied the effect of Lactobacillus acidophilus on diarrhea causing bacteria; E. coli, S. typhimurium, S. flexeneri and S. aureus. The Lactobacilli have been shown to possess inhibitory activity toward the growth of pathogenic bacteria. Our results revealed that the count of $E$. coli 0157:H7 decreased after 60 minutes from 240 to 17 cfu after mixing with $L B$-La5 (L1) and from 279 to $12 \mathrm{cfu}$ after mixing with $\angle B$ - ATCC 4356 (L2). As regarding the effect of L1 on control E-coli strain, the count of the latter deceased from 370 to 6 cfu after 60 minutes, while the count deceased from 350 to 11 cfu after 60 minutes with L2. In agreement with these results, Ogawa et al. [14] had documented the bactericidal activities of $L$. acidophilus against E.coli.

Abdel-Daim et al.[15] studied the probiotic potential and antagonistic activities of 32 Lactobacillus isolates against S. typhi and found that twelve Lactobacillus isolates could protect against $S$. typhi infections by interference with its growth and its virulence properties, such as adherence, invasion, and cytotoxicity. These results are nearly similar to the results of the current study since we found decease in the count of Salmonella (from 255 to18 cfu and from 222 to $20 \mathrm{cfu}$ ) after 60 minutes mixing with CFS of lactobacilli (LB-La5) and lactobacilli (LBATCC 4356), respectively.

This study revealed that the count of $S$. flexneri decreased to $10 \mathrm{cfu}$ after contact with Lactobacillus (LB-La5) for $60 \mathrm{~min}$, and to $15 \mathrm{cfu}$ after mixing with $L B$ - ATCC 4356 for 60 min. The study of Zhang et al.
[16] has documented that lactobacilli strongly inhibit the gastrointestinal pathogen Shigella.

The present study found that the count of S.aureus decreased from 340 to16 cfu and from 360 to $10 \mathrm{cfu}$, respectively after mixing with ( $L B$ $L a 5)$ and $L B$ - ATCC 4356, over one hour. Dicks and Botes [17] had reported that hydrogen peroxide produced by some strains of Lactobacilli, effectively inhibits Staphylococcus aureus, and L. acidophilus isolated from humans due to production of bacteriocin and non-bacteriocin antimicrobial substances which are active ( both in vitro and in vivo) tests against Gram-positive and Gram-negative pathogens. Similar results were previously obtained by the study of Bassyouni et al. [4], where 8 strains of Lactobacilli isolated from different dairy products showed antimicrobial effects on clinical isolates of E.coli, Staphylococcus spp., Salmonella spp. Micrococcus spp. using well diffusion method and with inhibition zone ranging from 13 to $25 \mathrm{~mm}$.

However, the result of this study disagree with Szymanski et al. [18] who has indicated that the effect of Lactobacilli was limited to the treatment of rotavirus induced diarrhea in children but not to the treatment of diarrhea of other etiology. Another study conducted by Szajewska et al. [19], reported also limitation of the therapeutic effects of probiotics on watery diarrhea and viral gastroenteritis but not against invasive bacterial diarrhea in children.

Synergistic effect of probiotics and antibiotics have been studied since 1990. Tomioka et al. [20] has investigated the effect of ofloxacin combined with Lactobacillus casei against Mycobacterium fortuitum induced infection in mice. They found that multiple injections of ofloxacin (subcutaneous or oral) in combination with a Lactobacillus casei preparation LC9018 (subcutaneous), in mice infected intravenously with Mycobacterium fortuitum led to the following; a marked delay in the incidence of spinning disease, a lowered incidence of gross renal lesions, and an increase in the rate of elimination of organisms from the kidneys. Their result in- 
dicates synergism in the therapeutic efficacy of the two agents. We have studied the effect Lactobacilli (L1and L2) in association with 20 antibiotics using disc diffusion method and found that both Lactobacilli significantly improve the antibacterial effect of tested antibiotics against ETEC, S. typhimurium and $S$. aureus, and $S$. flexeneri $(P<0.05)$. Similar results were reported by Ruiz et al. [21] using selected Lactobacillus fermentum strain L23 and L. rhamnosus strain L60 as an alternative treatment to prevent or treat urogenital infections. Their antimicrobial activity tests of L23 and L60 were performed by a disc diffusion method against 207 bacterial isolates from female presenting with urinary or genital infections. Their results showed $100 \%$ of the clinical isolates were susceptible to the antimicrobial substances produced by L23 and L60, and the selected lactobacilli produced larger inhibition zones when compared to several antibiotics commonly used for treating these infections. Synergistic interactions and indifferent interactions were recorded in 68.6\% and $31.4 \%$ of the cases, respectively, while no antagonistic interactions were observed. Also, Hussein et al. [22] found that $L$. acidophilusis specifically antagonizes $H$. pylori and enhances antibiotic therapy and its culture supernatants inhibit ulcer formation.

Fluoroquinolones are generally considered as the drugs of choice for the empirical treatment of diarrhea in adults, they are active against most of the common intestinal pathogens with excellent tissue and intracellular penetration, and achieve high fecal concentrations and have a good safety profile in adults [23]. Therefore, we investigated the effect of $L$. acidophilus with presence of ciprofloxacin against diarrhea causing bacteria in vitro. The MIC of ciprofloxacin alone against all tested strains was $15.625 \mu \mathrm{g} / \mathrm{ml}$, while when combined with both lactobacilli supernatants, the MIC decreased significantly to $0.488 \mu \mathrm{g} / \mathrm{ml}$ for ETEC, S. typhimurium and S.flexeneri and to $0.977 \mu \mathrm{g} / \mathrm{ml}$ for EHEC and S. aureus $(P=0.000)$. The overall results of this study are similar to results of Kaur and Sharma [24], which evaluated the synergism between conventional antibiotics and the cell-free supernatant (CFS) of vaginal Lactobacillus crispatus156 against $P$. aeruginosa MTCC 741 by checkerboard titrations. The used CFS succeeded to increase the activities of ciprofloxacin, moxifloxacin, and streptomycin, and it has decreased the MIC of ciprofloxacin by 30 times and MICs of both moxifloxacin and streptomycin by 8 times.

In conclusion, this study suggests that L. acidophilus can be used as an alternative therapy in treatment of different forms of infective bacterial gastroenteritis/diarrhea by its natural source in fermented milk and yoghurt or as mediations. The combined therapy of $L$. acidophilus with ciprofloxacin in treatment of infective gastroenteritis will help to decrease the required dose of ciprofloxacin and subsequent its potential side effects on intestinal bacterial flora.

\section{Conflict of Interest}

The authors declare that they have no conflict of interest

\section{References}

1. Kolader ME, Vinh $H_{\text {, }}$ TuyetPTN,Thompson C, WolbersM,Merson $\mathrm{L}$, et al.An oral preparation of Lactobacillus acidophilusfor the treatment of uncomplicated acute watery diarrhoea in Vietnamese children: study protocol for a multicentre, randomised, placebo-controlled trial. Trials 2013; 14:27

2. DuPont HL. Acute Infectious Diarrhea in Immunocompetent Adults. N Engl J Med 2014; 370:1532-1540

3. Allen SJ, Martinez EG, Gregorio GV, DansLF.Probiotics for treating acute infectious diarrhoea. Sao Paulo Med J 2011; 129, (3):185-185

4. Bassyouni.RH, Abdel-all WS, Fadl MG, Abdel-all S, kamel Z. Characterization of Lactic Acid Bacteria Isolated from Dairy Products in Egypt as a Probiotic. Life Sci J 2012; 9(4):2924-2933.

5. Shaukat A, Levitt MD, Taylor BC, MacDonald R, Shamliyan TA, Kane RL, Wilt TJ: Systematic review: effective management strategies for lactose intolerance. Ann Intern Med 2010; 152:797-803 
6. Johnston BC, Goldenberg JZ, Vandvik PO, Sun X, Guyatt GH: Probiotics for the prevention of pediatric antibiotic-associated diarrhea. Cochrane Database of Systematic Reviews (CDSR) 2011; 11: CD004827.

7. Fang SB, Lee HC, Hu JJ, Hou SY, Liu HL, Fang HW: Dosedependent effect of Lactobacillus rhamnosuson quantitative reduction of faecal rotavirus shedding in children.. J Trop Pediatr 2009; 55:297-301.

8. Phillip, S.,Mtshali, B.D., Maret du Toit. Identification and characterization of Lactobacillus florum strains isolated from South African grape and wine samples Int J Food Microbiol 2010; 153:106-113.

9. Godiosa O , Consignado C, Adrian, MD, Peña V Antoni, Jacalne DVM. In vitro study on the antibacterial activity of lactobacillus casei(commercial yakult drink) against four diarrhea causing organisms. Phil. J. Microbiol Infect Dis 1993; 22: 50-55.

10. Vuyst LD, Leroy F.Bacteriocin from lactic acid bacteria: Production, purification and food applications.J MolMicrobiolBiotechnol.2007; 13: 194-199.

11. Wolf CE, Gibbones WR. Improved method for quantification of the bacteriocinnicin. J Appl Bacteriol.1996; 80: 453-7.

12. Patton T, Barrett J,Brennan J,Moran N. Use of a spectrophotometric bioassay for determination of microbial sensitivity to manuka honey. J Microbiol Methods. 2006; 64(1): 84-95.

13. Clinical and Laboratory Standards Institute (CLSI). Performance standards for Antimicrobial Susceptibility Testing.22 Informational Supplement ed. CLSI 2012.document M100-S22. Wayne, PA.

14. Ogawa M, Shimizu K, Nomoto K, Tanaka R, Hamabata T, Yamasaki S, Takeda T, Takeda Y. Inhibition of in-vitro growth of Shiga toxin producing Escherichia coli 0157-H7 byprobiotic Lactobacillus strains due to production of lactic acid . Int J Food Microbiol.2001; 68: 135-40.

15. Abdel-Daim A, Hassouna N, Hafez M, Ashor MS, Aboulwafa MM. Antagonistic Activity of Lactobacilluslsolates againstSalmonella typhi In Vitro. Bio Med Research International 2013; 680605. doi: 10.1155/2013/680605

16. Zhang Y,Zhang L,Du M,Yi H,Guo C,TuoY, et al. Antimicrobial activity against Shigella sonnei and probiotic properties of wild lactobacilli from fermented food. Microbiol Res 2011; 167(1): 2731

17.Dicks LMN, Botes M. Probiotic lactic acid bacteria in the gastrointestinal tract: health benefits, safety and mode of action. Benef Microbes 2010; 1: 11-29.

18. Szymański H, Pejcz J, Jawień M, Chmielarczyk A, Strus M, Heczko PB. Treatment of acute infectious diarrhea in infants and children with a mixture of three Lactobacillus rhamnosus strains a randomized, double-blind, placebo-controlled trial. Aliment Pharmacol Ther 2006; 23(2): 247-53.

19. Szajewska H, Mrukowicz JZ. Use of probiotics in childrenwith acute diarrhea. Paediatr Drugs 2005; 7: 111-22.

20. Tomioka H, Sato K and Saito H.Effect of Ofloxacin Combined with Lactobacillus casei against Mycobacterium fortuitum
Infection Induced in Mice. Antimicrob Agents Chemother 1990; 632-636

21. Ruiz FO, Gerbaldo G,Asurmendi P, Pascual LM, Giordano W, Barberis IL.Antimicrobial activity, inhibition of urogenital pathogens, and synergistic interactions between lactobacillus strains. Curr Microbiol 2009; 59(5):497-501.

22. Hussein MZ, Gohar YM, Farag W. In-vitro Synergistic Actionof Curcumin and Lactobacillus acidophilus with Antibiotics against Helicobacter pylori. Egyptian J. Med. Micobiol 2007; 3: 521-32

23. Diniz-Santos DR, Silva LR, Silva N. Antibioticsfor the empirical treatment of acute infectious diarrhea in children. Braz J Infect Dis 2006;10(3):217-27

24. Kaur S, Sharma P. Protease-Sensitive Inhibitory Activity of Cellfree Supernatant of Lactobacillus crispatus 156 Synergizes with Ciprofloxacin, Moxifloxacin and Streptomycin Against Pseudomonas aeruginosa: An In Vitro Study. Probiotics Antimicrob Proteins 2015;7(2):172-80.

\section{Comment on this article:}

\section{8 in $8+\mathbf{S} P$}

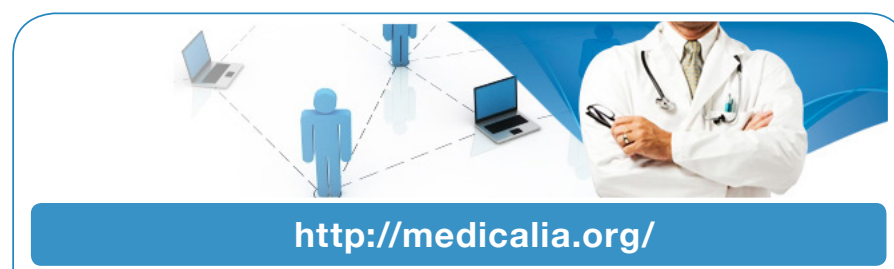

Where Doctors exchange clinical experiences, review their cases and share clinical knowledge. You can also access lots of medical publications for free. Join Now!

\section{Publish with iMedPub}

http://www.imed.pub

The Journal is an open access peer-reviewed journal that publishes scientific papers about all aspects of antimicrobials. The journal will publish original research articles, reviews, brief reports and case reports dealing with basic and clinical antibacterial agents, antiviral, antiprotozoals, antituberculuous, antifungal and antihelminthes agents. All manuscripts must be prepared in English, and are subject to a rigorous and fair peer-review process. Accepted papers will immediately appear online.

The journal aims to advance the knowledge, attitude and the research of chemotherapy in the Arabic world in cooperation with international, national scientific and public societies as well as research centers with similar aims and objectives. 